\title{
Reference values for gait temporal and loading symmetry of lower-limb amputees can help in refocusing rehabilitation targets
}

\author{
Andrea Giovanni Cutti ${ }^{*}$ Gennaro Verni, Gian Luca Migliore, Amedeo Amoresano and Michele Raggi \\ From Second World Congress hosted by the American Orthotic \& Prosthetic Association (AOPA) \\ Las Vegas, NV, USA. 06-09 September 2017
}

\begin{abstract}
Background: The literature suggests that optimal levels of gait symmetry might exist for lower-limb amputees. Not only these optimal values are unknown, but we also don't know typical symmetry ratios or which measures of symmetry are essential. Focusing on the symmetries of stance, step, first peak and impulse of the ground reaction force, the aim of this work was to answer to three methodological and three clinical questions. The methodological questions wanted to establish a minimum set of symmetry indexes to study and if there are limitations in their calculations. The clinical questions wanted to establish if typical levels of temporal and loading symmetry exist, and change with the level of amputation and prosthetic components.

Methods: Sixty traumatic, K3-K4 amputees were involved in the study: 12 transfemoral mechanical knee users (TFM), 25 C-leg knee users (TFC), and 23 transtibial amputees (TT). Ninety-two percent used the Ossur Variflex foot. Ten healthy subjects were also included. Ground reaction force from both feet were collected with the Novel Pedar-X. Symmetry indexes were calculated and statistically compared with regression analyses and non-parametric analysis of variance among subjects.

Results: Stance symmetry can be reported instead of step, but it cannot substitute impulse and first peak symmetry. The first peak cannot always be detected on all amputees. Statistically significant differences exist for stance symmetry among all groups, for impulse symmetry between TFM and TFC/T, for first peak symmetry between transfemoral amputees altogether and TT. Regarding impulse symmetry, 25\% of TFC and 43\% of TT had a higher impulse on the prosthetic side. Regarding first peak symmetry, 59\% of TF and 30\% of TT loaded more the prosthetic side.

Conclusions: Typical levels of symmetry for stance, impulse and first peak change with the level of amputation and componentry. Indications exist that C-leg and energy-storage-and-return feet can improve symmetry. Results are suggestive of two mechanisms related to sound side knee osteoarthritis: increased impulse for TF and increased first peak for $\Pi$. These results can be useful in clinics to set rehabilitation targets, understand the advancements of a patient during gait retraining, compare and chose components and possibly rehabilitation programs.
\end{abstract}

Keywords: Gait, Ground reaction force, Symmetry, Rehabilitation, Amputees, Prosthesis, Osteoarthritis, C-leg, Microprocessor controlled knees, Energy storage and return feet

\footnotetext{
* Correspondence: ag.cutti@inail.it

INAIL Prosthetic Center, Via Rabuina 14, 40054 Vigorso di Budrio, BO, Italy
}

(c) The Author(s). 2018 Open Access This article is distributed under the terms of the Creative Commons Attribution 4.0 International License (http://creativecommons.org/licenses/by/4.0/), which permits unrestricted use, distribution, and reproduction in any medium, provided you give appropriate credit to the original author(s) and the source, provide a link to the Creative Commons license, and indicate if changes were made. The Creative Commons Public Domain Dedication waiver (http://creativecommons.org/publicdomain/zero/1.0/) applies to the data made available in this article, unless otherwise stated. 


\section{Background}

Lower-limb amputees tend to walk asymmetrically when looking at gait temporal and loading parameters, with more time spent and load exerted on the intact limb [1-9]. Temporal asymmetry is typically measured based on step or stance duration; loading asymmetry based the magnitude of the first peak of the vertical ground reaction force (GRF), and the impulse of GRF [2, 3, 6, 10].

Temporal and loading asymmetries were associated to several comorbidities [5]: increased falls [11], osteoarthritis of the sound limb [10,12-15], osteoporosis of the contralateral limb $[15,16]$, back pain $[17-20]$. In addition, walking in public with noticeable asymmetries attracts the general attention [21], which can be very uncomfortable for some prosthesis users. With this background, it is not surprising that a common, almost unquestioned [22], goal for rehabilitation is to regain a symmetric walking $[9,23]$.

However, the literature does not clearly indicate that striving for perfect symmetry is really and always the best option. Already in 1998, Winter \& Sienko [1] stated that "human system with major structural asymmetries in the neuromuscular skeletal system cannot be optimal when gait is symmetrical. Rather, a new non-symmetrical optimal is probably being sought by the amputee within the constraints of his residual system and the mechanics of his prosthesis". Later in 2005, Schmid and co-workers [3] compared the center of pressure trajectories under the sound and prosthetic foot of transfemoral amputees and concluded that the longer stance on the sound side can be ascribed to the greater ability of the sound leg to advance the step and maintain balance until the prosthetic limb can sustain the body weight. Hof et al. [4] corroborated this explanation in the theoretical framework of the "extrapolated center of mass" [24], concluding that stance time asymmetry is a "sensible adaptation" of experienced transfemoral amputees to improve stability during walking, to overcome the missing lateral ankle strategy of prosthetic feet. More recently, Adamczyk \& Kuo [8], with a theoretical and experimental approach involving transtibial amputees, concluded that "some asymmetry may be unavoidable in cases of unilateral limb loss" due to the reduced ankle plantar flexion of the ankle, with direct consequences on stance duration, greater collision work at the sound side, greater work overall, and increased peak force at loading response [25-27]. Imposing symmetry can actually be detrimental, as also observed by [27, 28].

The evidences from the literature, therefore, indicate that optimal symmetry ratios might exist, to obtain a compromise among stability, forward progression, preservation of body structures and perception of a "normal and symmetric biped locomotion" [21]. Unfortunately, at present not only optimal symmetry ratios are unknown, but we also don't know typical symmetry ratios or which measures of symmetry are essential and which are redundant.
In our opinion, 3 methodological and 3 clinical questions should be answered to clarify these open issues. The methodological questions are:

- Q1: do all amputees show the typical M-shaped pattern of the GRF [29], with presence and appropriate timing of its two peaks? In case of a negative answer, the measure of loading symmetry based on the first peak of GRF will be restrict to patients presenting the M-shaped pattern;

- Q2: can we limit the study of temporal symmetry to stance, leaving out step symmetry? We will give a positive answer if stance and step symmetries are very strongly correlated for all amputees, with a coefficient of determination $R^{2}>0.64$ [30];

- Q3: can we further limit the study of gait symmetry to just stance symmetry, leaving out loading symmetry, whose measure requires more cumbersome and expensive equipment? We will give a positive answer if stance symmetry is very strongly correlated $\left(R^{2}>0.64\right)$ with the symmetry of the first peak and impulse of GRF.

\section{The clinical questions are:}

- Q4: does gait symmetry depend on the level of amputation? In case of a positive answer, typical ranges of symmetry should be established, which can be used to understand how far a new patient is from well adapted prosthesis users in terms of percentiles;

- Q5: do advanced prosthetic components improve temporal and loading symmetry? In particular, do $\mathrm{C}$-leg users have better results than mechanical knee users of the same mobility level?

- Q6: is it always true that amputees overload the sound side both in terms of first peak and impulse of GRF, thus contributing to the development of osteoarthritis?

Unfortunately, at present it is difficult to answer to these questions based on the available literature, because there are no studies that considered, at the same time 1) both temporal and loading asymmetries, 2) both transfemoral and transtibial amputees treated at the same prosthetic \& rehabilitation center, 3) mechanical and electronic knees, 4) energy-storage-and-return feet instead of the SACH (Solid-Ankle Cushion-Heel) foot. Moreover, the number of patients included is typically limited to 8 , both for studies on transtibial and transfemoral amputees. Finally, no studies addressed the correlation between temporal and loading parameters.

The aim of this study was to overcome these limitations and answer to questions Q1-Q6 on three groups of 
well-adapted, traumatic, K3-K4 amputees: transfemoral amputees using a restricted set of mechanical knees (TFM), transfemoral amputees using the C-leg (TFC), transtibial amputees using energy-storage-and-return feet (TT). A additional group of healthy control subjects ("Controls" in short), was also included to highlight general trends.

\section{Methods}

\section{Subjects}

Sixty K3-K4 lower-limb amputees participated in the study after signing an informed consent: 12 mechanical knee users (TFM, $46 \pm 10$ y.o.), 25 C-leg users (TFC, $48 \pm$ 13 y.o), 23 transtibial amputees (TT, $44 \pm 14$ y.o.), with no statistically significant differences in term of age (ANOVA, $p>0.62)$. Ten controls were also included ( $28 \pm 2$ y.o.). All amputees had completed a 3-week, intense gait training program at the same specialized prosthetic \& rehabilitation center, with the support of the same rehabilitation team. The clinical center has ISO 9001 treatment pathways for amputees and provides over 800 transfemoral and 1200 transtibial prostheses every year. Following training, all patients had been successfully using their prostheses for at least 1 month at the time of testing.

The components provided to patients are summarized in Table 1. Almost $92 \%$ of patients used either the Variflex or Variflex LP foot. Mechanical knees were selected to match the activity level of the C-leg, and are consistent with knees selected for comparison with the C-leg in previous studies [31, 32].

\section{Measurements}

After standing still for $10 \mathrm{~s}$, subjects walked along a long indoor hall at self-selected speed, that was noted. During this trial, the GRF was measured on each side through instrumented insoles (Pedar-X, Novel, D), sampling at $100 \mathrm{~Hz}[33,34]$.

\section{Data processing}

For each subject, GRF data were export to MATLAB. Based on the $10 \mathrm{~s}$ ' orthostatic posture, body weight was calculated. Assuming a foot-floor contact threshold at $10 \%$ body weight, we detected heel-strike and toe-off

Table 1 Prosthetic components used and associated quantities

\begin{tabular}{llll}
\hline & TFM & TFC & TT \\
\hline Foot & Variflex LP: 10 & Variflex LP: 25 & Variflex: 18 \\
& 1C40: 2 & & Variflex LP: 2 \\
& & & Truestep: 1 \\
& & & Esprit: 1 \\
& & & 1C40: 1 \\
Knee & TotalKnee 2100: 5 & C-leg: 25 & \\
& 3R60: 2 & & \\
& Mauch: 2 & & \\
\hline
\end{tabular}

events for the two sides. We isolated the steady state condition by considering the central 10 strides.

\section{Calculation of temporal symmetry}

For each stride, we calculated the step and stance duration. Then, for each couple of consecutive sound-affected gait cycles, we calculated the following indexes of symmetry:

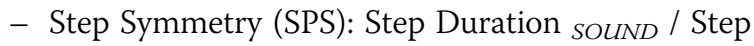 Duration AFFECTED \\ - Stance Symmetry (SNS): Stance Duration SOUND / Stance Duration AFFECTED}

For Controls, ratios were right over left side. A value of 1 represents perfect symmetry. For each index of symmetry, we calculated the subject's median value over the trial. Finally, we obtained the distribution of the median values for the two indexes over TFM, TFC, TT and Controls.

\section{Calculation of loading symmetry}

For each gait cycle, the integral over the stance period of GRF was calculated, i.e. the impulse of $G R F$, as previously reported by [2]. Then, for each couple of consecutive sound-affected gait cycles, we calculated the index of symmetry:

$$
\begin{aligned}
& \text { - Impulse Symmetry (IMS): Impulse souND / } \\
& \text { Impulse } \text { AFFECTED }^{\text {Im }}
\end{aligned}
$$

A value of 1 represents perfect symmetry. Right over left side was used for Controls.

Afterward, the GRF profile of each gait cycle was checked to verify the presence of the first peak within the $0-40 \%$ of the gait cycle, and of a second peak within the $60-100 \%$. Subjects reporting both peaks in more than half of the trials formed the "Two-Peaks" subgroup.

For the subjects in Two-Peaks we operated as follows. For each couple of consecutive sound-affected gait cycles, we calculated the following index:

$$
\begin{aligned}
& \text { - First Peak Symmetry (P1S): First peak sOUND / } \\
& \text { First peak } \text { AFFECTED }
\end{aligned}
$$

P1S provides a measure of peak force asymmetry at loading response, while IMS provides a measure of the asymmetry in cyclic loading. These are two different mechanism of osteoarthritis development [10, 35-37].

For each index of symmetry, we calculated the subject's median value over the trial. Finally, we obtained the distribution of the median values for the two indexes over TFM, TFC, TT and Controls. 


\section{Statistical analysis}

The distribution of the four indexes of symmetry (SPS, SNS, IMS and P1S) was checked for normality within each group (TFM, TFC, TT and Controls) and over all subjects, both visually with the Normal Probability Plot and with the Lilliefors test. This last failed for SPS ${ }_{\text {TFM }}$ and $\mathrm{P} 1 \mathrm{~S}_{\mathrm{TT}}$ and there were doubts about IMS in general.

The relationship between SNS and the three indexes SPS, IMS and P1S was evaluated with regression methods with the MATLAB Curve Fitting Toolbox. The strength of the relationship was primarily evaluated in terms of $R^{2}$. This statistical parameter, multiplied by 100 , is usually interpreted as the variance of " $y$ " accounted for by " $x$ ", where in this case " $y$ " is SPS or IMS or P1S, and " $x$ " is SNS. In addition, the root-mean-square error (RMSE) of the residuals was also reported.

Distributions were reported in terms of median and interquartile range [3], with box plots. For each symmetry index, the Kruskal-Wallis test $(\alpha=0.05)$ was adopted to check for overall statistically significant differences among TFM, TFC, TT and Controls. In identifying pairwise differences, the Tukey-Kramer "HSD" correction was applied within the MATLAB "multcompare" function.

\section{Results}

Gait speed was compared among TFM $(1.12 \pm 0.13 \mathrm{~m} / \mathrm{s})$, TFC $(1.17 \pm 0.12 \mathrm{~m} / \mathrm{s})$, TT $(1.23 \pm 0.19 \mathrm{~m} / \mathrm{s})$ and Controls $(1.41 \pm 0.21 \mathrm{~m} / \mathrm{s})$. ANOVA did not show statistically significant differences among amputees $(p=0.14)$, but only between Controls and amputees $(p=0.0005)$.

Further results are reported hereinafter based on their relevance for questions Q1-Q6.

\section{Question Q1}

Figure 1 reports the number of subjects in subgroups Two-Peaks, which decreases from TT (20/23), to TFM $(7 / 12)$ to TFC (10/25). The number of TFC with nonstandard GRF is remarkably high (60\%); these patients report a consistent "alternative" pattern (example provided in Fig. 1b). Based on these results, the answer to Q1 was negative and the calculation of the symmetry index P1S was restricted to the subjects in Two-Peaks.

\section{Question Q2}

Figure 2 reports the regression analysis for SPS vs SNS considering the whole set of patients and Controls ("ALL" in brief). $R^{2}$ and RMSE values for each group

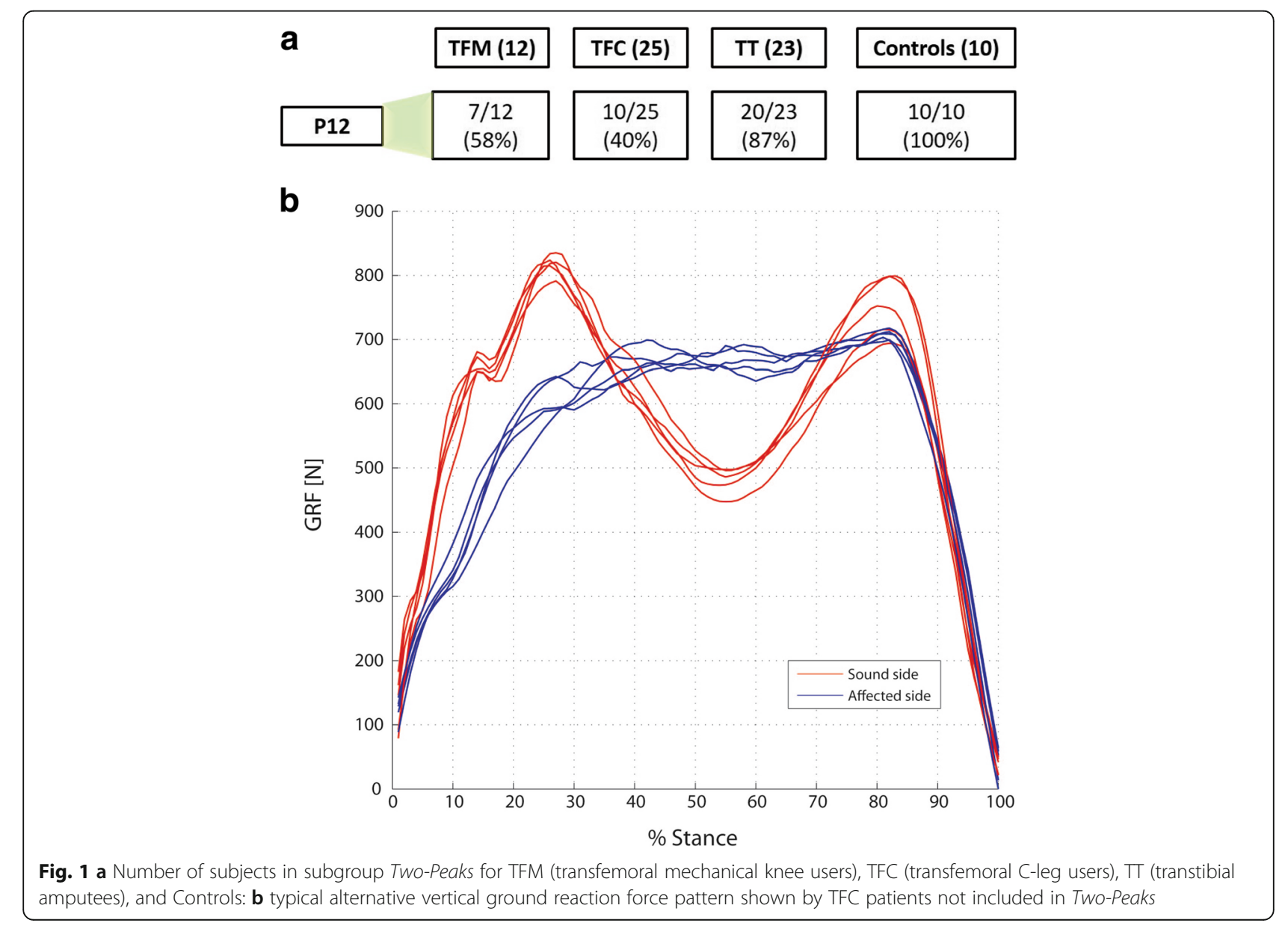




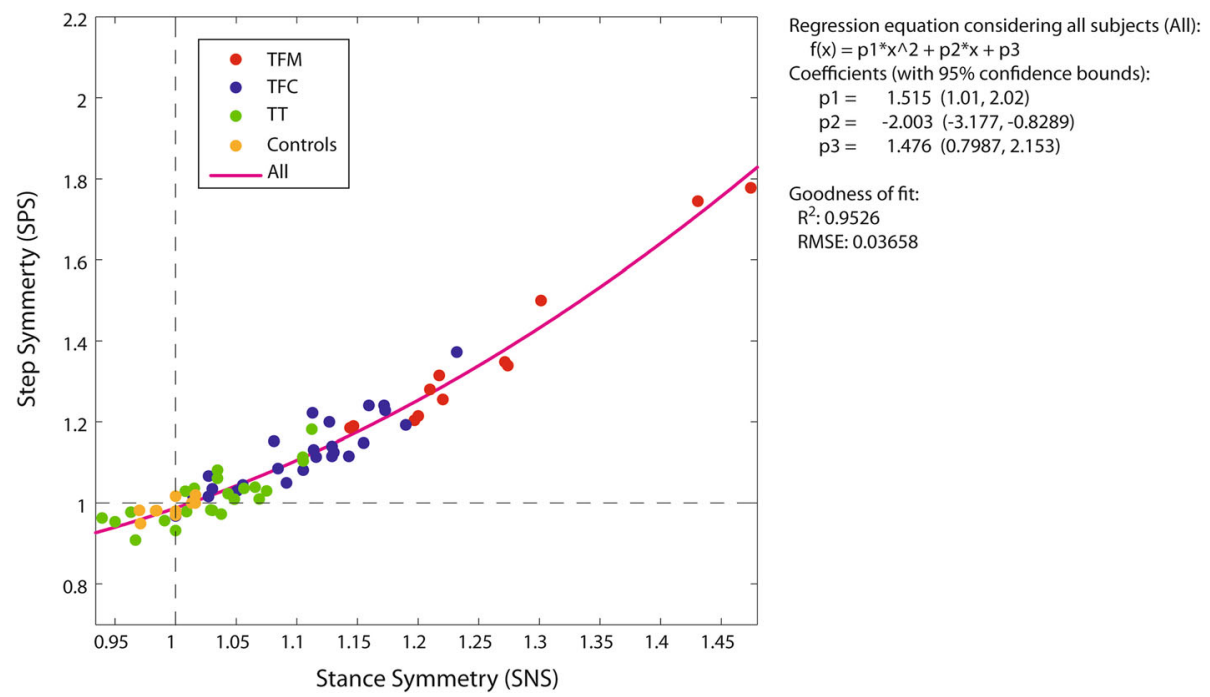

Fig. 2 Step symmetry index (SPS) vs Stance symmetry index (SNS). Each dot represents one subject. Subjects of the same group feature the same color (see legend in the plot). The purple parabolic line is the regression line for ALL subjects together. The equation of the fitting is reported on the right, with the fitting quality parameters $R^{2}$ (coefficient of determination) and RMSE (Root Mean Squared Error)

(TFM, TFC, TT, Controls) and ALL are reported in Table 2. $R^{2}$ was at least 0.70 for all amputees, with RMSE $<0.042$. Therefore, the answer to Q2 was positive and only SPS was further considered.

\section{Question Q3}

Figure $3 \mathrm{a}$ and $\mathrm{b}$ report the regression analysis for IMS vs SNS and P1S vs SNS, respectively, for ALL. $R^{2}$ and RMSE values for each group (TFM, TFC, TT, Controls) and ALL are reported in Table 2. For IMS vs SNS, $R^{2}$ was lower than 0.64 for TT, with RMSE $>0.128$. For P1S vs SNS, $R^{2}$ was lower than 0.2 for all amputees. Therefore, the answer to Q3 was negative and IMS, P1S and SPS were separately considered in all subsequent analyses.

Table 2 Quality of fit of the regressions for step (SPS), impulse (IMS) and first peak symmetry (P1S) indexes vs stance symmetry index (SNS)

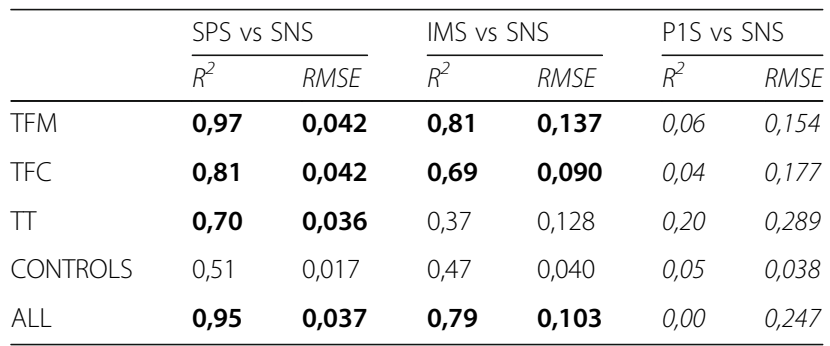

The coefficient of determination $\left(R^{2}\right)$, and the Root Mean Squared Error (RMSE) are reported for every group (TFM transfemoral mechanical knee users, TFC transfemoral C-leg users, $\Pi$ transtibial amputees, Controls), and for all subjects altogether (ALL). Bold: $R^{2}>0.64$, Regular: $0.36<R^{2}<0.64$, Italic: $R^{2}<0.36$ [30]

\section{Questions Q4-Q6}

Figures $4 \mathrm{a}, 5 \mathrm{a}$ and $6 \mathrm{a}$ report the distribution of SNS, IMS and P1S for TFC, TFC, TT and Controls. Numerical values are reported in Table 3.

For SNS and IMS, the Kruskal-Wallis test showed statistically significant differences among the medians of the groups $(p<0.0001)$ (Figs. $4 \mathrm{~b}$ and $5 \mathrm{~b})$. The pairwise analyses for:

- SNS (Fig. 4c) showed that all amputee groups are different among each other, supporting a positive answer for Q4 and Q5;

- IMS (Fig. 5c) showed a statistically significant difference between TFM and all other groups, with all TFM values $>1$ as opposed to TFC and TT. This supports a partially positive answer to $Q 4$, a positive answer to Q5 and a negative answer to Q6.

For P1S, the Kruskal-Wallis test reported a statistically significant difference in the medians among groups $(p=0.0443)$ (Fig. 6b). The pairwise comparison did not show differences (Fig. 6c). This is a very possible situation for three reasons:

- the Kruskal-Wallis and pairwise comparisons try to negate different hypotheses;

- we applied a quite conservative multiple comparison strategy (HSD);

- the statistical power is reduced by the decreased number of transfemoral amputees (TF) within Two-Peaks. 

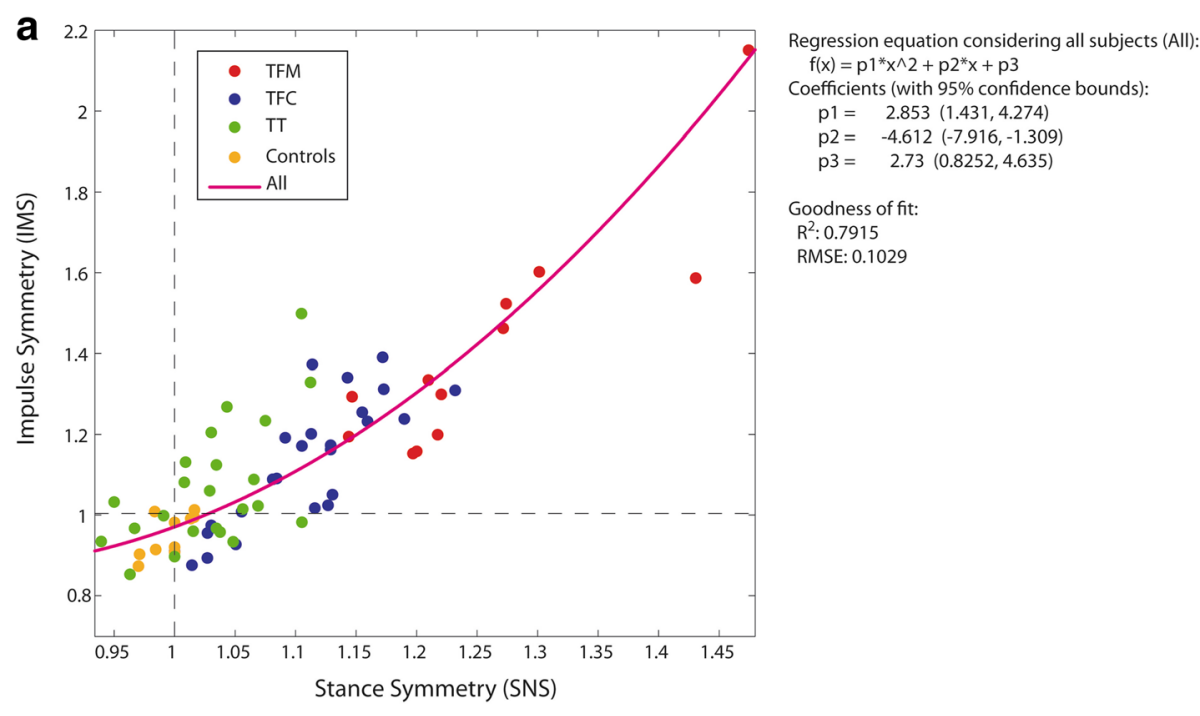

b

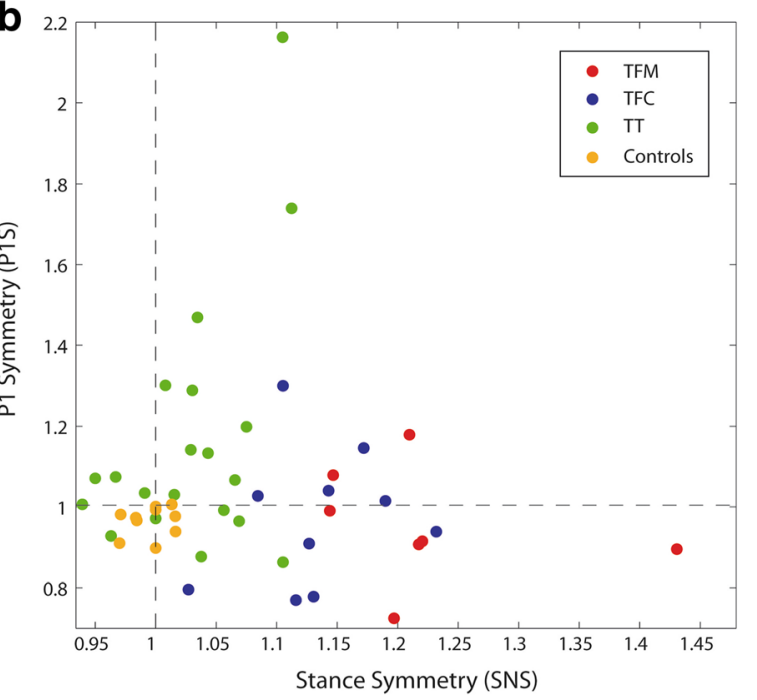

Fig. 3 a Impulse symmetry index (IMS) vs Stance symmetry index (SNS) and b First peak symmetry index (P1S) vs SNS. Each dot represents a subject. Subjects of the same group feature the same color (see legend in the plot). In (a), the purple parabolic line is the regression line for ALL subjects together. The equation of the fitting is reported on the right, with the fitting quality parameters $R^{2}$ (coefficient of determination) and RMSE (Root Mean Squared Error). No valid regression was found for P1S vs SNS. TFM: transfemoral mechanical knee users, TFC: transfemoral C-leg users, TT: transtibial amputees

For this reason, we grouped subjects per level of amputation (TFM and TFC together), and results are reported in Fig. 7a. The Kruskal-Wallis test now shows a stronger significance among groups $(p=0.0186)$ and the pairwise analysis shows a statistically significant difference between TF and TT. The variability in P1S is much higher in amputees than in Controls (Bartlett's test for equal variances, $p=0.001)$. These results support a negative answer to Q6.

\section{Discussion}

In this study, we addressed three methodological and three clinical questions regarding the temporal and loading symmetry of transfemoral amputees (both mechanical and C-leg users) and transtibial amputees, to support in the development of more targeted rehabilitation goals, that are particularly needed $[9,38]$.

As a general consideration, the self-selected walking speed was not statistically different among amputees, despite a slight increase in the median from TFM, to TFC, to TT toward Controls. Absolute values compare well with previously reported data $[2,7,39]$.

For the sake of clarity, results are discussed below for each question, in comparison with the available literature whenever possible.

\section{Question Q1}

Question Q1 asked if all amputees show the typical M-shaped pattern of the GRF, with presence and 


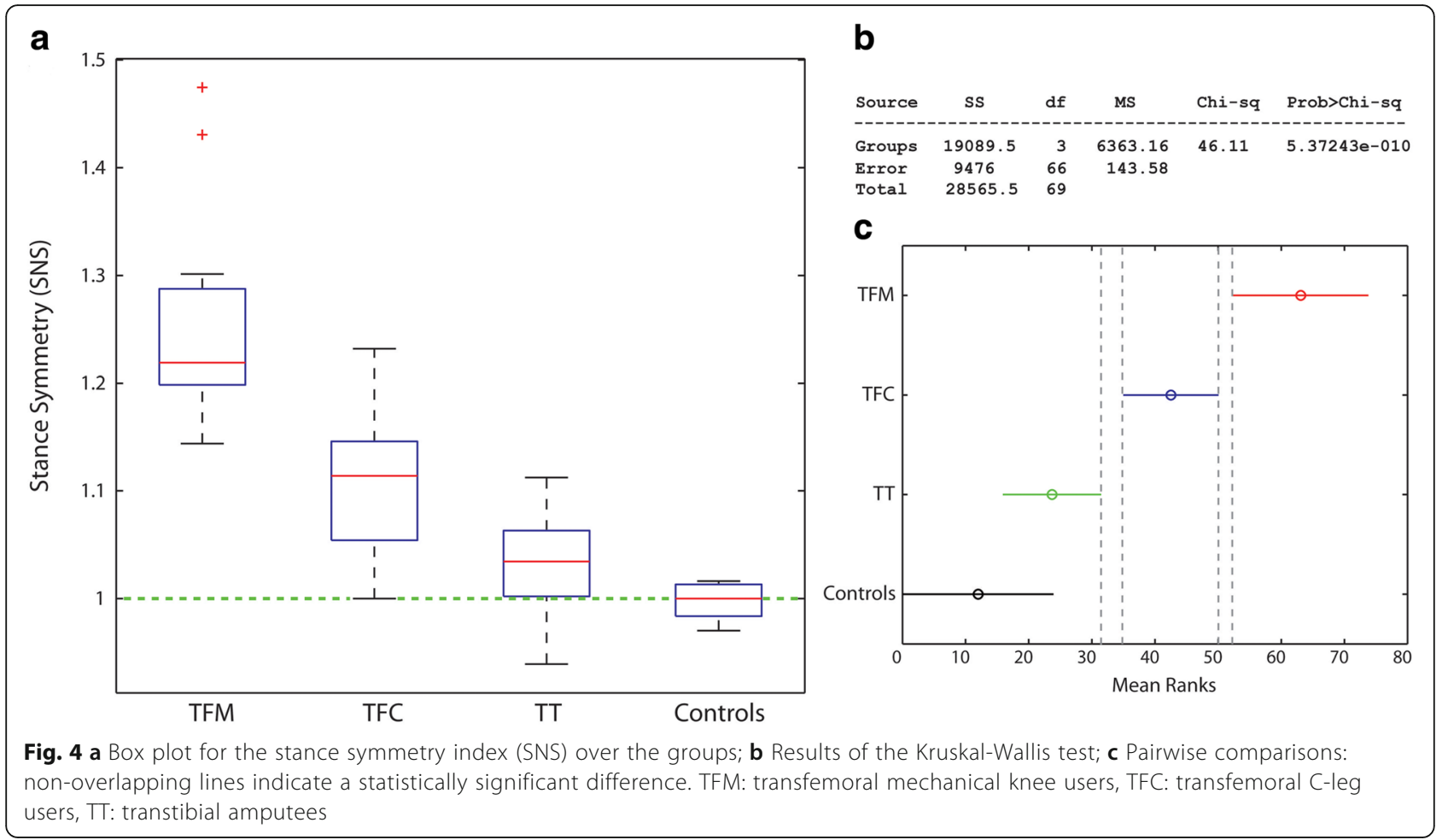

appropriate timing of its two peaks. Results support a negative answer.

As previously noted, this is particularly evident for TFC, who presented a consistent "alternative" pattern: after a steep rise (initial contact/loading response), GRF shows a further (almost) monotonical increase (midstance), after which it drops (terminal stance/pre-swing). TFM falling out of Two-Peaks did not present this pattern, and were typically not included in Two-Peaks due to a delayed P1 after $40 \%$ of the stance phase. Since no kinematic and

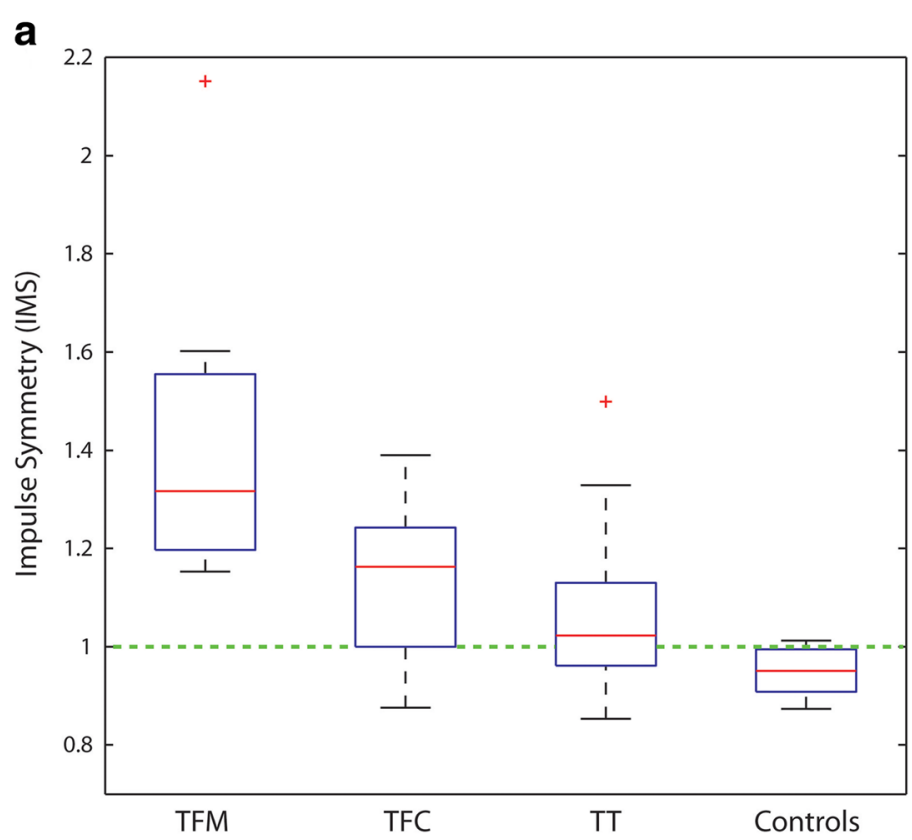

\section{b}

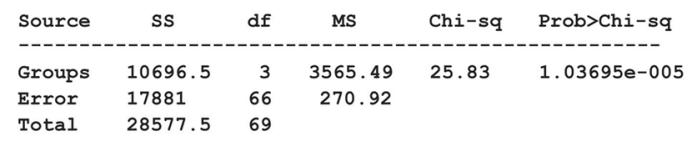

Total $28577.5 \quad 69$

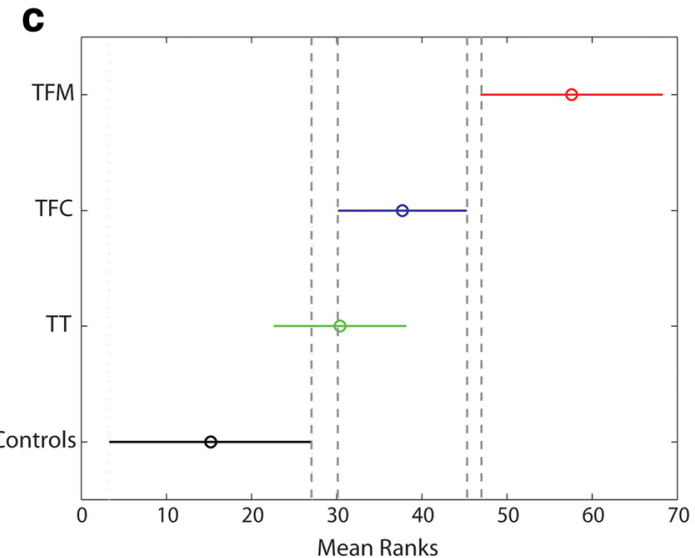

Fig. 5 a Box plot for the impulse symmetry index (IMS) over the groups; b Results of the Kruskal-Wallis test; c Pairwise comparisons: non-overlapping lines indicate a statistically significant difference. TFM: transfemoral mechanical knee users, TFC: transfemoral C-leg users, T: transtibial amputees 


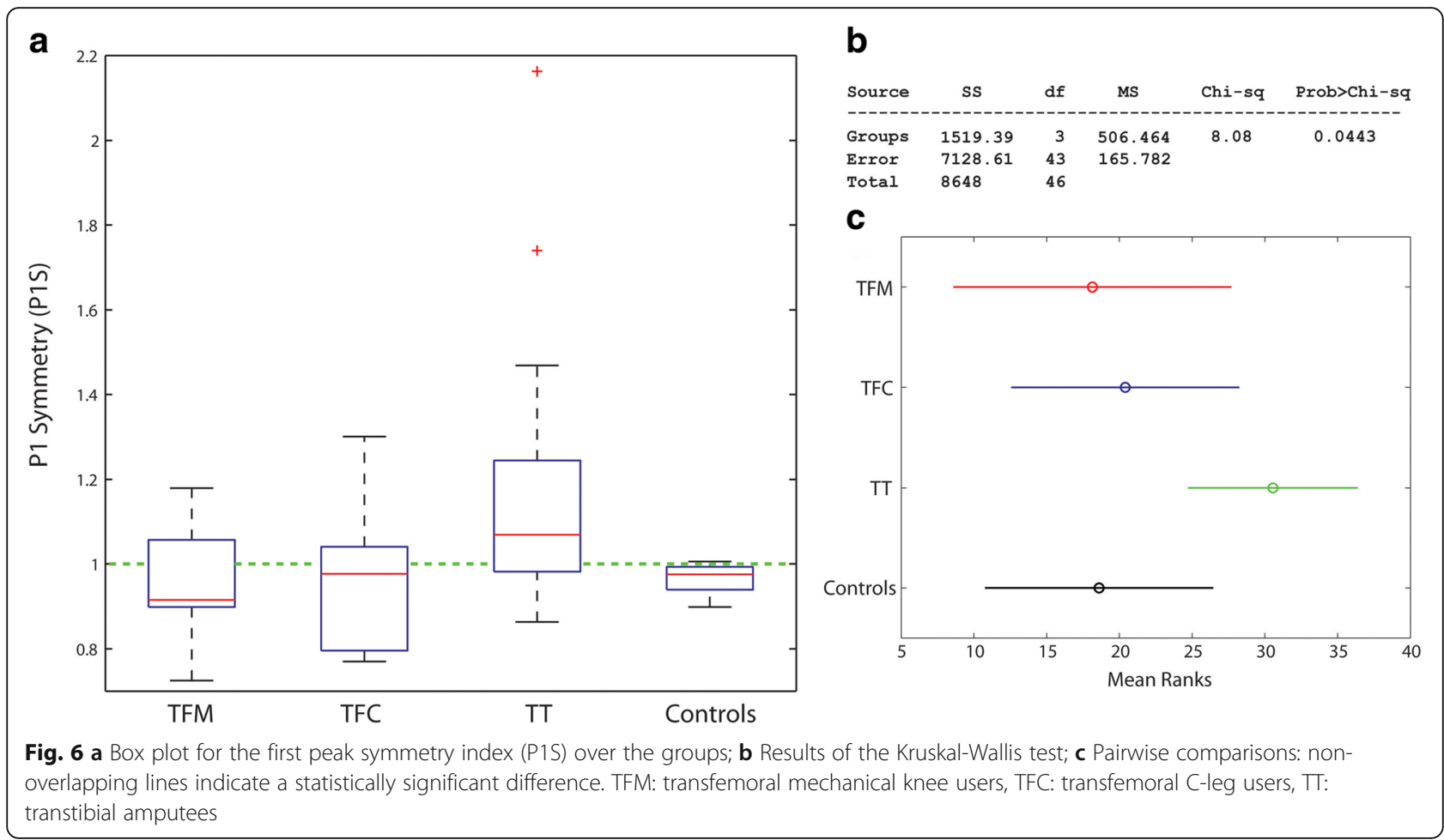

kinetic data were collected, we can just speculate that this TFC pattern is the combined effect of:

- the Variflex behavior, with strong energy storage in loading response $[25,26]$;

- C-leg knee flexion in loading response [40];

- the confidence gained by this group of amputees on the capacity of the C-leg to sustain them at heel-strike and loading response, with no need to force extension.

The ultimate effect for this pattern is a "soft landing" on the prosthetic side, which might increase comfort [32]. These speculations require future experimental confirmations, but match well with previous evidences that only a fraction of transfemoral amputees can fully rely on C-leg stability despite knee flexion during early stance $[32,40]$. This might be the effect of a specialized rehabilitation.

\section{Question Q2}

Question Q2 asked if we can limit the study of temporal symmetry to stance leaving out step symmetry. Results support a positive answer.

The regression of SPS vs SNS for each group and for ALL was quadratic, with excellent fits.

SNS explained from 70 to $97 \%$ of the variance in SPS data in amputees $\left(R^{2}\right.$, as reported in Table 2). Even for Controls, who feature a very small peak-to-peak SNS (.97 to 1.01), the explained SPS variance is $50 \%$ with a RMSE as small as 0.017 .

Table 3 Numerical values for the indexes of symmetry SNS (stance), IMS (impulse) and P1S (first peak)

\begin{tabular}{|c|c|c|c|c|c|c|c|c|c|c|c|c|}
\hline & \multicolumn{4}{|l|}{ SNS } & \multicolumn{4}{|l|}{ IMS } & \multicolumn{4}{|l|}{ P1S } \\
\hline & Median & 25th & 75th & IQR & Median & 25th & 75th & IQR & Median & 25th & 75th & IQR \\
\hline TFM & 1,22 & 1,20 & 1,29 & 0,09 & 1,32 & 1,20 & 1,55 & 0,36 & 0,91 & 0,90 & 1,06 & 0,16 \\
\hline TFC & 1,11 & 1,05 & 1,15 & 0,09 & 1,16 & 1,00 & 1,24 & 0,24 & 0,98 & 0,80 & 1,04 & 0,24 \\
\hline TF & & & & & & & & & 0,94 & 0,87 & 1,05 & 0,18 \\
\hline Tा & 1,03 & 1,00 & 1,06 & 0,06 & 1,02 & 0,96 & 1,13 & 0,17 & 1,07 & 0,98 & 1,24 & 0,26 \\
\hline CONTROLS & 1,02 & 0,98 & 1,01 & 0,03 & 0,95 & 0,91 & 0,99 & 0,09 & 0,98 & 0,94 & 0,99 & 0,05 \\
\hline
\end{tabular}

For each group, the median is reported together with the 25th, 75th and interquartile range (IQR). TFM transfemoral mechanical knee users, TFC transfemoral Cleg users, $T F$ transfemoral, $T$ transtibial amputees 


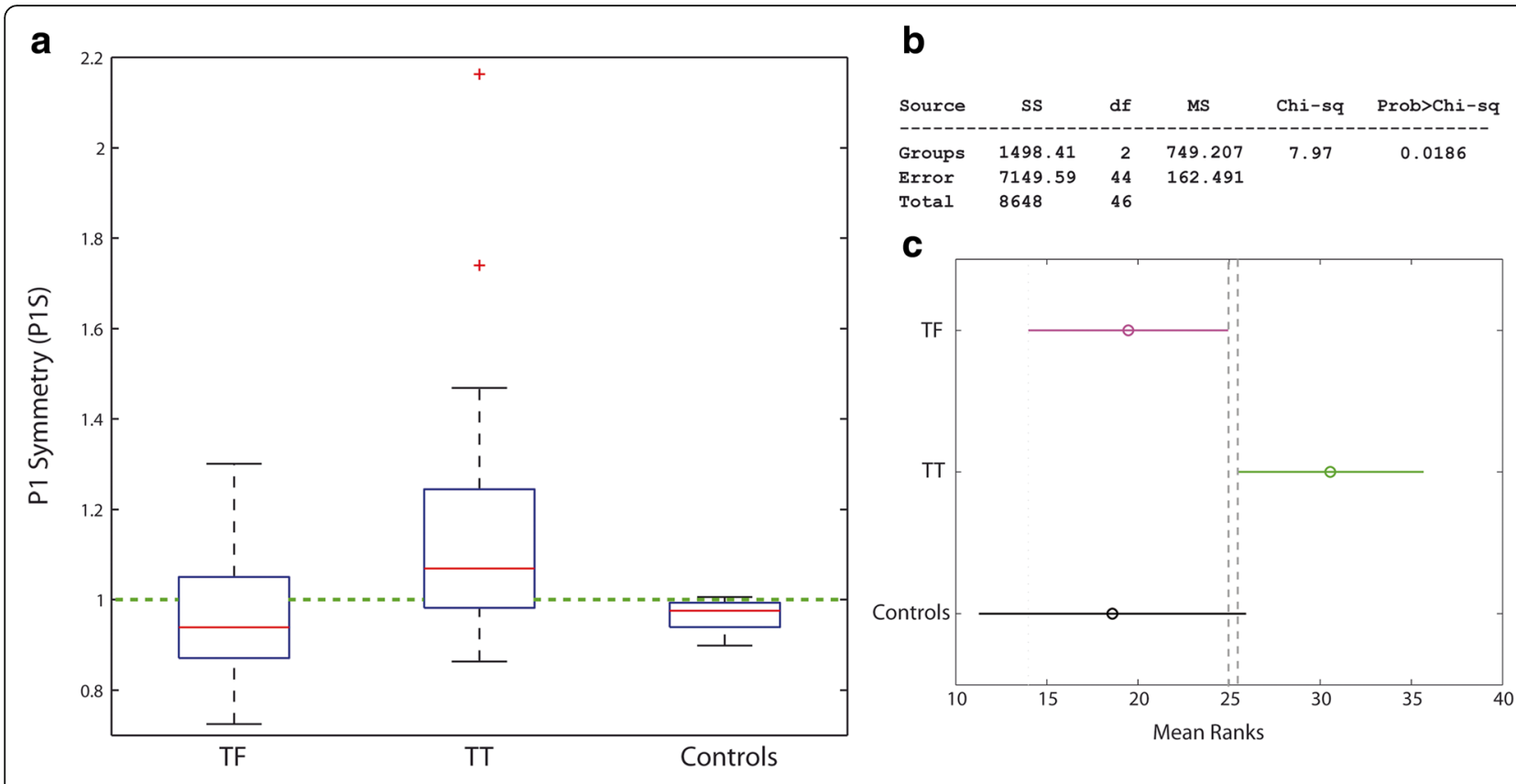

Fig. 7 a Box plot for the first peak symmetry index (P1S) after grouping all transfemoral amputee together (TF); b Results of the Kruskal-Wallis test; c Pairwise comparisons: non-overlapping lines indicate a statistically significant difference. TF: transfemoral amputees, TT: transtibial amputees

This is the first time that the SPS vs SNS regression is reported in the literature and that a quadratic relationship is described. The nonlinear fit is not surprising, because SPS is non-linearly related to the interplay of 1) the sounds and affected side stance durations and 2) the two double support durations. The quadratic fit stresses the importance of stance time symmetry, since it influences step asymmetry by a factor 2 .

\section{Question Q3}

Question Q3 asked if the study of gait symmetry can be limited to just stance temporal symmetry, leaving out loading symmetry. Results support a negative answer.

When IMS vs SNS was examined considering the full set of subjects, a quadratic fit emerged: SNS explained as much as $79 \%$ of the variance in IMS. This is the first time this relationship is examined and reported. Since IMS is the integral of GRF over the stance phase, it is not surprising that IMS and SNS are related: a high stance time asymmetry is a leading factor for a high impulse asymmetry. However, GRF magnitude does not linearly increase with time, and has a shape which can differ between the sound and affected side. When all these elements become part of a ratio, it is not surprising that the relation between IMS and SNS can be non-linear.

This conclusion is valid for TFM and TFC at group level too, given the $R^{2}>0.64$. However, this is just partially true for TT, because $R^{2}$ decreases to 0.37 and the RMSE is high (0.128): reporting SNS and not IMS can be misleading. This different evidence for TT can be ascribed to two factors only:

- The improvement in SNS asymmetry (1.03, IQR 0.06) compared to TFM (1.22, IQR 0.09) and TFC (1.11, IQR 0.09) (Table 3);

- A greater asymmetry in GRF magnitude between sides. This is supported by the evidences for P1S, as reported in Fig. 7. Further discussions are postponed to Q6 below.

An adequate regression for P1S vs SNS was not found for none of the groups and ALL: the two indexes must measure different construct and therefore they must be separately reported.

\section{Question Q4}

Question Q4 asked if gait symmetry depends on the level of amputation. Results support a positive answer.

With reference to SNS, all amputee groups had statistically different median values. All TF spend more time on the sound side: TFM have the highest asymmetry (median asymmetry of $22 \%$ ), which is twofold the TFC's (11\%). As can be seen in Fig. 4, this is also true for $75 \%$ of TT, which means that $1 / 4$ of TT do spend more time on the affected side. This was never clearly reported in the literature. The TT asymmetry (3\%) is 4 times less than TFC. Controls, in median, have a perfect symmetry, with a IQR of just $3 \%$. 
The SNS median for TFC (1.11) compares well with the median SNS that can be calculated from the results reported in [3] (1.09). Furthermore, our results can be compared with the study of Nolan et al. [2], that involved 4 transfemoral and 4 transtibial amputees using a single hinge knee and a $\mathrm{SACH}$ foot. Once appropriately converted to our indexes, Nolan's results are reported in Table 4. Results, can also be compared with Bateni et al. [41], which reported a mean stance asymmetry for $\mathrm{TT}$ of about $7 \%$ (calculated as the ratio of the mean between sides). Compared to these studies, our SNS values are lower. In particular, $63 \%$ of TT and $20 \%$ of TFC have a SNS lower than $\pm 5 \%$, which makes them unperceived by others as "impaired" walkers with regard to temporal symmetry [21]. This is not surprising given the different prosthetic components used and the fact that our patients followed a specialized rehabilitation training. Our SNS results for TT are also in very good agreement with results reported by Jarvis et al. [38] for young veterans (median 1.04, IQR $=0.03$ ). For TFC, our SNS is higher (1.11 compared to 0.98$)$ but the IQR is much smaller (0.09 compared to 0.20$)$. This remarks that the training for transfemoral amputees is more challenging.

When looking at IMS, the TFM median was statistically different from TFC and TT: TFM asymmetry is twice that of TFC and 16 times TT's. The comparison with Nolan et al. [2] is striking: our TFM had an impulse asymmetry which is half Nolan's; for TT it is 10 times less. This result points, again, in the direction of the benefits of energy-storage-and-return feet and more advanced knees. Improvement in loading asymmetry with energy-storage-and-return feet and feet with improved roll-over shape has been previously reported in [25, 27, 42], and match well with simulation studies [8].

Finally, P1S results show statistically significant differences between TF and TT (Fig. 7). About 59\% of TF have a higher peak on the prosthetic side. Our results agree with Castro et al., which did not report an increased peak GRF on the sound side, but rather an increase in the GRF impulse. TT clearly show an asymmetric loading with higher values for the sound side (70\% of patients), but 3 times less

Table 4 Results from Nolan et al. [2], converted to the indexes of symmetry used in this study. SNS (stance), IMS (impulse) and P1S (first peak)

\begin{tabular}{llll}
\hline & SNS & IMS & P1S \\
\hline TFM & 1,27 & 1,69 & 1,22 \\
$\pi$ & 1,05 & 1,36 & 1,25 \\
CONTROLS & 1,03 & 1,08 & 1,08 \\
\hline
\end{tabular}

Having named $N$ the indexes in [2], the new values follow from this equation: $\mathrm{New}=(2+N) /(2-N)$

TFM transfemoral mechanical knee users, $\Pi$ transtibial amputees than that reported by Nolan and co-workers. As previously reported, it is reasonable to ascribe this improvement to the use of energy-storage-and-return feet compared to $\mathrm{SACH}[27,43]$.

\section{Question Q5}

Question Q5 asked if advanced prosthetic components improve temporal and loading symmetry, and if C-leg users have better results than mechanical knee users of the same mobility level. Results support a positive answer.

Results have been partially discussed while addressing Q1 and Q4 and can be summarized stating that TFC were statistically different from TFM for SNS and IMS. Results for IMS bring TFC to undistinguishable results to TT.

Also, the C-leg in combination with Variflex triggers a new GRF pattern that possibly ensures an increased comfort during walking (Question Q1). This requires further experimental confirmations.

Petersen et al. [44] have previously reported about SNS in C-leg users compared to TFM. However, that study was not able to prove a statistically significant improvement but just a trend, probably due to the small number of subjects included (5) with different amputation etiologies. Our results confirm that trend, with statistically significant differences. More generally, a considerable body of knowledge is available about the positive effects of the C-leg on amputees' mobility [31, 45-47], gait kinematic [32-40], kinetic [39] and step-length symmetry [32]. Our findings match well with this general trend toward improved symmetries.

As discussed in Q4, the comparison of the literature with our results for TT suggests a possible positive effect of energy-storage-and-return feet in comparison with $\mathrm{SACH}$, for all the indexes of symmetry.

\section{Question Q6}

Question Q6 asked if it is always true that amputees overload the sound side both in terms of first peak and impulse of GRF, thus contributing to the development of osteoarthritis. Results support a negative answer.

As previously discussed about Q4, if we focus on IMS, $100 \%$ of TFM overload the sound side. This percentage decreases to $75 \%$ of TFC and $57 \%$ of TT. If we look at P1S, 41\% TF load more the sound side. However, this percentage rises to $70 \%$ for TT. Based on these different percentages of TT and TF for IMS and P1S, it could be argued that two different mechanisms might be related to knee osteoarthritis for the two groups: peak overload for TT (measured by P1S), and extended duration of force action (impulse) for TF (measured by IMS). Given the higher prevalence of knee osteoarthritis in TF compared to TT $[5,10]$, it might be speculated that the second mechanism is more detrimental than the first. 


\section{Conclusions}

In the Introduction, we posed three methodological and three clinical questions regarding the gait temporal and loading symmetry of lower-limb amputees. Based on the results collected on traumatic, K3-K4, transfemoral (mechanical knees and C-leg users) and transtibial patients successfully fit and trained in using their prosthesis, we can answer as follows.

The three methodological questions wanted to establish a minimum set of symmetry indexes to study and if there are limitations in their calculations. First, the first peak of the vertical ground reaction force at loading response cannot be clearly identified in all amputees, and the calculation of its index of symmetry was limited to patients with the typical M-Shaped pattern of the ground reaction force. Second, the analysis of temporal symmetry can be limited to stance, leaving out step symmetry. Third, stance, impulse and first peak symmetries should be separately reported.

The three clinical questions wanted to establish if "typical" levels of temporal and loading symmetry exist and change with the level of amputation and prosthetic components. First, the symmetries of stance, impulse and first peak are all influenced by the level of amputation. In particular, the time spent on the sound side decreases significantly from transfemoral mechanical knee users, to C-leg users, to transtibial patients. The impulse on the sound side decreases significantly from mechanical knee users to C-leg and transtibial patients. Transtibial patients have a higher first peak at loading response on their sound side, while most transfemoral patients do not. Second, advanced prosthetic component seem to positively influence the temporal and loading symmetry. In particular, the C-leg in combination with the Variflex foot improves stance, impulse symmetry and for about $60 \%$ of patients smooths the first peak at loading response. About $20 \%$ of C-leg users have a stance asymmetry which is below the level of perceived impaired gait, compared to $0 \%$ of mechanical knee users. For transtibial patients, comparisons of our results with the literature point toward an improvement of all indexes of symmetry, possibly due to the use of energy-storage-and-return feet instead of SACH feet. Third, it is not always true that amputees overload the sound side. Percentagewise, transfemoral amputees tend to overload the sound side with increased impulse, while TT with increased peak GRF. This might be suggestive of two separate mechanisms for the onset of knee osteoarthritis.

We think that our results can be exploited in the clinical routine. First, clinicians can use our results to set reasonable targets for rehabilitation. Specifically, they can compare the level of symmetry of a new patient with the ranges provided, and put the patient's performance and advancements during rehabilitation in perspective. Moreover, technical and healthcare professionals might use our findings to compare the effect of different prosthetic components and potentially the effect of different rehabilitation programs. Second, it is often required by payers (e.g. insurances, public healthcare services, or patients), to justify the use of advanced prosthetic components. We think that our results support the use of C-leg and energy-storage-and-return feet on K3-K4 traumatic patients: thanks to the improvement in temporal and loading symmetry compared to mechanical knees and $\mathrm{SACH}$ foot, these components can potentially have a positive effect on the asymmetry-related comorbidities analyzed in the Introduction and decrease social stigma. Further research is required to extend these results to other groups of patients, such as K2 and non-traumatic amputees. Finally, our results might suggest possible strategies to mitigate knee osteoarthritis of the sound side. Pending further research, transfemoral amputees might take advantage of prosthetic components with an improved knee-foot coordination to specifically tackle stance time asymmetry. Transtibial patients might benefit from improved socket construction that does not limit knee extension, and prosthetic feet with improved push-off, roll-over shape and range of motion to reduce the first peak at loading response.

\begin{abstract}
Abbreviations
GRF: Vertical component of the ground reaction force; IMS: Impulse symmetry index; P1S: Symmetry index of the first peak of the ground reaction force; SNS: Stance duration symmetry index; SPS: Step duration symmetry index; TF: Transfemoral amputees; TFC: Transfemoral amputees using a C-leg knee (Ottobock, D); TFM: Transfemoral amputees using a mechanical knee; TT: Transtibial amputees
\end{abstract}

\section{Funding}

This research was conducted with internal institutional funds of INAIL. The publication cost of this article was funded by the American Orthotic \& Prosthetic Association (AOPA).

\section{Availability of data and materials}

All data generated or analysed during this study are included in this published article.

\section{About this supplement}

This article has been published as part of Journal of NeuroEngineering and Rehabilitation Volume 15 Supplement 1, 2018: Advancements in Prosthetics and Orthotics: Selected articles from the Second World Congress hosted by the American Orthotic \& Prosthetic Association (AOPA). The full contents of the supplement are available online at https://jneuroengrehab.biomedcentral.com/ articles/supplements/volume-15-supplement-1.

Authors' contributions

AGC, MR and GV designed the experiment. MR and AGC collected and processed the data. All Authors contributed to data analysis and manuscript preparation. All authors read and approved the final manuscript.

Ethics approval and consent to participate

The Centro Protesi institutional scientific committee approved the study.

Consent for publication

Not applicable.

Competing interests

The authors declare that they have no competing interests. 


\section{Publisher's Note}

Springer Nature remains neutral with regard to jurisdictional claims in published maps and institutional affiliations.

Published: 5 September 2018

\section{References}

1. Winter DA, Sienko SE. Biomechanics of below-knee amputee gait. J Biomech. 1988;21(5):361-7.

2. Nolan L, Wit A, Dudziñski K, Lees A, Lake M, Wychowañski M. Adjustments in gait symmetry with walking speed in trans-femoral and trans-tibial amputees. Gait Posture. 2003;17(2):142-51.

3. Schmid M, Beltrami G, Zambarbieri D, Verni G. Centre of pressure displacements in trans-femoral amputees during gait. Gait Posture. 2005; 21(3):255-62.

4. Hof AL, van Bockel RM, Schoppen T, Postema K. Control of lateral balance in walking. Experimental findings in normal subjects and above-knee amputees. Gait Posture. 2007;25(2):250-8.

5. Gailey R, Allen K, Castles J, Kucharik J, Roeder M. Review of secondary physical conditions associated with lower-limb amputation and long-term prosthesis use. J Rehabil Res Dev. 2008;45(1):15-29.

6. Castro MP, Soares D, Mendes E, Machado L. Plantar pressures and ground reaction forces during walking of individuals with unilateral transfemoral amputation. PM\&R. 2014;6(8):698-707.

7. Wezenberg D, Cutti AG, Bruno A, Houdijk H. Differentiation between solidankle cushioned heel and energy storage and return prosthetic foot based on step-to-step transition cost. J Rehabil Res Dev. 2014;51(10):1579-90.

8. Adamczyk PG, Kuo AD. Mechanisms of gait asymmetry due to push-off deficiency in unilateral amputees. IEEE Trans Neural Syst Rehabil Eng. 2015; 23(5):776-85.

9. Highsmith MJ, Andrews CR, Millman C, Fuller A, Kahle JT, Klenow TD, Lewis KL, Bradley RC, Orriola JJ. Gait training interventions for lower extremity amputees: a systematic literature review. Technol Innov. 2016;18(2-3):99-113.

10. Morgenroth DC, Gellhorn AC, Suri P. Osteoarthritis in the disabled population: a mechanical perspective. PM\&R. 2012:4(5 Suppl):S20-7.

11. Vanicek N, Strike S, McNaughton L, Polman R. Gait patterns in transtibial amputee fallers vs. non-fallers: biomechanical differences during level walking. Gait Posture. 2009;29(3):415-20.

12. Norvell DC, Czerniecki JM, Reiber GE, Maynard C, Pecoraro JA, Weiss NS. The prevalence of knee pain and symptomatic knee osteoarthritis among veteran traumatic amputees and nonamputees. Arch Phys Med Rehabil. 2005;86:487-93

13. Struyf PA, van Heugten CM, Hitters MW, Smeets RJ. The prevalence of osteoarthritis of the intact hip and knee among traumatic leg amputees. Arch Phys Med Rehabil. 2009;90:440-6.

14. Lemaire ED, Fisher FR. Osteoarthritis and elderly amputee gait. Arch Phys Med Rehabil. 1994;75(10):1094-9.

15. Burke MJ, Roman V, Wright V. Bone and joint changes in lower limb amputees. Ann Rheum Dis. 1978;37(3):252-4.

16. Rush PJ, Wong JS, Kirsh J, Devlin M. Osteopenia in patients with above knee amputation. Arch Phys Med Rehabil. 1994;75(1):112-5.

17. Shojaei I, Hendershot BD, Wolf EJ, Bazrgari B. Persons with unilateral transfemoral amputation experience larger spinal loads during level-ground walking compared to able-bodied individuals. Clin Biomech (Bristol, Avon). 2016;32:157-63. https://doi.org/10.1016/j.clinbiomech.2015.11.018.

18. Yoder AJ, Petrella AJ, Silverman AK. Trunk-pelvis motion, joint loads, and muscle forces during walking with a transtibial amputation. Gait Posture. 2015;41(3):757-62.

19. Russell Esposito E, Wilken JM. The relationship between pelvis-trunk coordination and low back pain in individuals with transfemoral amputations. Gait Posture. 2014:40(4):640-6.

20. Rabuffetti M, Recalcati M, Ferrarin M. Trans-femoral amputee gait: socketpelvis constraints and compensation strategies. Prosthetics Orthot Int. 2005; 29(2):183-92.

21. Handžić I, Reed KB. Perception of gait patterns that deviate from normal and symmetric biped locomotion. Front Psychol. 2015;6:199.

22. Marinakis GN. Interlimb symmetry of traumatic unilateral transtibial amputees wearing two different prosthetic feet in the early rehabilitation stage. J Rehabil Res Dev. 2004;41(4):581-90.

23. Esquenazi A. Gait analysis in lower-limb amputation and prosthetic rehabilitation. Phys Med Rehabil Clin N Am. 2014;25(1):153-67.
24. Hof AL. The 'extrapolated center of mass' concept suggests a simple control of balance in walking. Hum Mov Sci. 2008;27(1):112-25.

25. Powers MC, Torburn L, Perry J, Ayyappa E. Influence of prosthetic foot design on sound limb loading in adults with unilateral below-knee amputations. Arch Phys Med Rehabil. 1994;75(7):825-9.

26. Snyder RD, Powers CM, Fountain C, Perry J. The effect of five prosthetic feet on the gait and loading of the sound limb in dysvascular below-knee amputees. J Rehabil Res Dev. 1995;32:309-15.

27. Hansen AH, Meier MR, Sessoms PH, Childress DS. The effects of prosthetic foot roll-over shape arc length on the gait of trans-tibial prosthesis users. Prosthetics Orthot Int. 2006;30(3):286-99.

28. Gard SA. Use of quantitative gait analysis for the evaluation of prosthetic walking performance. J Prosthet Orthot. 2006;18(6):P93-P104.

29. Perry J, Burnfield J. Gait analysis: normal and pathological function: Thorofare: SALCK Inc: 2010.

30. Evans JD. Straightforward statistics for the behavioral sciences. Pacific Grove: Brooks/Cole Publishing; 1996

31. Cutti AG, Lettieri E, Del Maestro M, Radaelli G, Luchetti M, Verni G, Masella C. Stratified cost-utility analysis of C-leg versus mechanical knees: findings from an Italian sample of transfemoral amputees. Prosthetics Orthot Int. 2017:41(3):227-36

32. Segal AD, Orendurff MS, Klute GK, McDowell ML, Pecoraro JA, Shofer J, Czerniecki JM. Kinematic and kinetic comparisons of transfemoral amputee gait using C-leg and Mauch SNS prosthetic knees. J Rehabil Res Dev. 2006; 43(7):857-70.

33. Putti AB, Arnold GP, Cochrane L, Abboud RJ. The Pedar in-shoe system: repeatability and normal pressure values. Gait Posture. 2007;25(3):401-5.

34. Hurkmans HL, Bussmann JB, Benda E, Verhaar JA, Stam HJ. Accuracy and repeatability of the Pedar Mobile system in long-term vertical force measurements. Gait Posture. 2006;23(1):118-25.

35. Dekel S, Weissman SL. Joint changes after overuse and peak overloading of rabbit knees in vivo. Acta Orthop Scand. 1978;49(6):519-28.

36. Guilak F. Biomechanical factors in osteoarthritis. Best Pract Res Clin Rheumatol. 2011;25(6):815-23.

37. Buckwalter JA, Anderson DD, Brown TD, Tochigi Y, Martin JA. The roles of mechanical stresses in the pathogenesis of osteoarthritis: implications for treatment of joint injuries. Cartilage. 2013:4(4):286-94.

38. Jarvis HL, Bennett AN, Twiste M, Phillip RD, Etherington J, Baker R. Temporal spatial and metabolic measures of walking in highly functional individuals with lower limb amputations. Arch Phys Med Rehabil. 2016;98(7):1389-99.

39. Kaufman KR, Frittoli S, Frigo CA. Gait asymmetry of transfemoral amputees using mechanical and microprocessor-controlled prosthetic knees. Clin Biomech (Bristol, Avon). 2012;27(5):460-5.

40. Kaufman KR, Levine JA, Brey RH, Iverson BK, McCrady SK, Padgett DJ, Joyner MJ. Gait and balance of transfemoral amputees using passive mechanical and microprocessor-controlled prosthetic knees. Gait Posture. 2007;26(4):489-93.

41. Bateni $\mathrm{H}$, Olney SJ. Kinematic and kinetic variations of below-knee amputee gait. J Prosthet Orthot. 2004;14(1):2-10.

42. Underwood HA, Tokuno CD, Eng JJ. A comparison of two prosthetic feet on the multi-joint and multi-plane kinetic gait compensations in individuals with a unilateral trans-tibial amputation. Clin Biomech (Bristol, Avon). 2004;19:609-16.

43. Lehmann JF, Price R, Boswell-Bessette S, Dralle A, Questad K, deLateur BJ. Comprehensive analysis of energy storing prosthetic feet: flex foot and Seattle foot versus standard SACH foot. Arch Phys Med Rehabil. 1993;74(11):1225-31.

44. Petersen $\mathrm{AO}$, Comins J, Alkjær T. Assessment of gait symmetry in transfemoral amputees using C-leg compared with 3R60 prosthetic knees. J Prosthet Orthot. 2010:22(2):106-12

45. Hahn A, Lang M. Effects of mobility grade, age, and etiology on functional benefit and safety of subjects evaluated in more than 1200 C-leg trial fittings in Germany. J Prosthet Orthot. 2015;27(3):86-94.

46. Hafner BJ, Willingham LL, Buell NC, Allyn KJ, Smith DG. Evaluation of function, performance, and preference as transfemoral amputees transition from mechanical to microprocessor control of the prosthetic knee. Arch Phys Med Rehabil. 2007;88(2):207-17. Erratum in: Arch Phys Med Rehabil. 2007 Apr:88(4):544

47. Highsmith MJ, Kahle JT, Bongiorni DR, Sutton BS, Groer S, Kaufman KR. Safety, energy efficiency, and cost efficacy of the C-leg for transfemoral amputees: a review of the literature. Prosthetics Orthot Int. 2010;34(4):362-77. 